\title{
Factores sociodemográficos asociados a los lazos parentales en estudiantes de Lima Norte
}

\author{
Sociodemographic factors associated with parental ties in students of \\ northern Lima
}

Juana Quispe-Rodriguez ${ }^{1}$
Heber Flores-Acuña

\begin{abstract}
Resumen
Objetivo: determinar la relación entre los lazos parentales y las variables sociodemográficas en los estudiantes de un centro pre-universitario. Materiales y métodos: estudio de alcance correlacional y diseño no experimental de corte transversal. La muestra estuvo conformada por 187 estudiantes de un centro pre-universitario de Lima norte. Las variables principales fueron: lazos parentales y factores sociodemográficos. Se realizó el análisis de datos a través del software estadístico STATA 14. Resultados se encontró relación entre la dimensión de sobreprotección padre y cuidador primario $(\mathrm{p}=0.00)$, al igual que sobreprotección madre $(\mathrm{p}=0.008)$ y cuidador primario. Asimismo, la dimensión sobreprotección madre se relacionó con el lugar de nacimiento $(\mathrm{p}=0.001)$. Se obtuvieron valores altos en la dimensión de sobreprotección de la madre $(93.0 \%)$ y el padre (82.3\%). Conclusión: los padres biológicos son percibidos como más sobreprotectores que otros cuidadores primarios. Esta relación podría disminuir la autonomía, la capacidad de decisión y adaptación, efectos característicos de la sobreprotección. La relación entre la sobreprotección de la madre y el lugar de nacimiento puede estar relacionada a factores psicosociales y culturales que deben ser más estudiados en próximos estudios. Se sugiere promover la intervención psicológica sistemática para padres e hijos en el ámbito comunitario y educativo.
\end{abstract}

Palabras clave: Crianza; Cuidadores; Datos demográficos; Relaciones Padres-Hijos; Estudiantes (Fuente: DeCS).

\begin{abstract}
Objective: to determine the relationship between parental ties and sociodemographic variables in the students of a preuniversity center. Materials and methods: study of correlational scope and non-experimental cross-sectional design. The sample consisted of 187 students from a pre-university center in northern Lima. The main variables were: Parental bonds and sociodemographic factors. Data analysis was performed through statistical software STATA 14. Results: a relationship was found between the parent overprotection and primary caregiver dimension $(\mathrm{p}=0.00)$, as well as mother overprotection $(\mathrm{p}=0.008)$ and primary caregiver. Likewise, the mother overprotection dimension was related to the place of birth $(\mathrm{p}=0.001)$. The highest values of the dimensions of parental ties were recorded in There is also significant relationship between parental bonds in the dimension of overprotection of the mother with place of birth (93.63\%). High values were obtained in the dimension of overprotection of the mother (93.05\%) and the father (82.35\%). Conclusions: biological parents are perceived as more overprotective than other primary caregivers. This relationship could diminish the autonomy, the capacity of decision and adaptation, characteristic effects of overprotection. The relationship between the overprotection of the mother and the place of birth may be related to psychosocial and cultural factors that should be more studied in future studies. It is suggested to promote systematic psychological intervention for parents and children in the community and educational environment.
\end{abstract}

Key words: Parenting; Caregivers; Demographic data; Parent- Child Relations; Students (Source: DeCS).

Para citar:

Quispe J, Flores H. Factores sociodemográficos asociados a los lazos parentales en estudiantes de Lima norte. CASUS. 2018;3(2):97-102.

\footnotetext{
${ }^{1}$ Hospital Cayetano Heredia. Bachiller en Psicología.

${ }^{2}$ Hospital Nacional Hipólito Unanue. Bachiller en Psicología

Correo electrónico: juanitaucss@gmail.com
}

Fecha de recepción: 28-05-18

Fecha de envío a pares: 29-05-18

Fecha de aprobación por pares: 26-08-18

Fecha de aceptación: 28-08-18 


\section{INTRODUCCIÓN}

En la población juvenil se evidencian diversas problemáticas psicológicas que tienen su origen en el vínculo o estilo de crianza que perciben y reciben de sus padres $(1,2)$. Se hipotetiza que a la base de ellas se podría encontrar que no han establecido una adecuada relación con sus padres o cuidadores primarios. En este sentido, en el Perú se ha identificado que el $30.09 \%$ de jóvenes de 18 a 25 años perciben que sus padres ejercen un vínculo de control sin afecto hacia ellos $\mathrm{y}$ consecuentemente presentan niveles muy altos de dependencia emocional en sus relaciones afectivas (3).

Este vínculo o también denominado lazo parental es definido como la percepción que tiene el individuo hasta los 16 años de aquellos comportamientos y actitudes establecidos por las figuras parentales durante su crianza (4). Es decir, un sujeto en desarrollo dependerá del vínculo que haya formado con un adulto para determinar su posterior estabilidad o fracaso emocional (5). Por ello, es importante la figura de apego con sus cuidadores primarios o padres durante la infancia y la adolescencia que servirán como base para su desarrollo socioafectivo. Por el contrario, un vínculo deficiente, sin afecto, o de constricción puede producir lazos parentales de bajo cuidado o de sobreprotección (6). Es así, que los padres pueden desarrollar una relación de sobreprotección hacia los hijos, en la cual el padre ejerce un estilo de crianza de control para proteger al menor de posibles situaciones de riesgo (7).

Según la perspectiva del "aprendizaje social" diversos factores como la ubicación geográfica, cultural y el lugar donde reside la familia, podrían generar un estilo de crianza de sobreprotección, caracterizado por el padre proveedor y la madre cuidadora (8 ). De esta manera, se comprende que los lazos parentales pueden variar en función de las características sociodemográficas del adolescente y su estructura familiar (9).

En el Perú, se ha registrado que los adolescentes que provienen de Lima Metropolitana y el Callao, que en su mayoría estudian o han culminado la educación de nivel secundaria y con problemas económicos familiares producto de su situación laboral experimentan diversas problemáticas como la irritabilidad, conducta disocial (tendencia psicopática), conducta suicida (pensamiento e ideación suicida), tendencia a la violencia, episodio depresivo y uso de sustancias adictivas (10).

Frente a estos problemas y a la presencia de estresores psicosociales que afectan su salud mental, señalaron como principal factor protector a la cohesión familiar que involucra sentimientos de apoyo, identidad y estilos de crianza percibidos por los hijos (10). Por lo tanto, resulta importante investigar variables como el lugar de nacimiento, personas encargadas de la crianza de los hijos, número de integrantes en el hogar y otros que aporten a la mayor comprensión de los estilos o lazos parentales percibidos (11). Es por ello, que se hace necesario conocer qué factores sociodemográficos se asocian a la percepción de los lazos parentales sobre los progenitores o cuidadores primarios. Puesto que, a nivel nacional son escasas las contribuciones que profundizan en estos factores, el estudio tuvo como objetivo determinar la relación entre factores sociodemográficos y los lazos parentales en estudiantes de un centro pre-universitario de Lima norte.

\section{MATERIALES Y MÉTODOS}

Estudio de tipo correlacional de corte transversal y enfoque cuantitativo. Se realizó un censo que evaluó a la totalidad de la población y estuvo conformado por 187 estudiantes de 16 a 25 años de edad pertenecientes a un Centro pre-universitario de Lima norte. Las variables principales fueron: lazos parentales y factores sociodemográficos.

Lazos parentales es la percepción que tiene el hijo sobre los comportamientos y actitudes establecidos por las figuras parentales que pueden determinar un vínculo óptimo de confianza, apoyo y comunicación; o por el contrario, la carencia de vínculo genera inestabilidad, miedo y ansiedad (4). La variable fue medida a través del instrumento de Lazos Parentales (12). Esta variable es de naturaleza cualitativa dicotómica y está compuesta 
por dos dimensiones que se interpretan considerando el sexo en padre y madre. La dimensión cuidado que comprende el afecto e indiferencia se categoriza en el nivel alto, (40-48) para las mujeres y (36-48) para los varones, en la cual existen manifestaciones de un vínculo óptimo y actitudes cariñosas hacia los hijos. Así mismo el nivel bajo, (22-39) para las mujeres y (15-35) para los varones, se caracteriza por un vínculo débil y escasas manifestaciones de afecto. La dimensión de sobreprotección que está comprendida por el excesivo control y afecto desmedido se categoriza en nivel alto, (29-47) para las mujeres y (27-43) para los varones, relacionado al contacto excesivo y limitación de la autonomía de los hijos. El nivel bajo, (14-28) para las mujeres y (13-26) para los varones, está determinado por un vínculo ausente o débil caracterizado por presentar actitudes de indiferencia y negligencia.

Las variables sociodemográficas fueron recolectadas a través de una ficha sociodemográfica, en relación a las mismas se consideraron: la variable cuidador primario padre medida a través de la pregunta: ¿durante la mayor parte de su vida el rol de su padre lo ha cumplido?, de naturaleza politómica nominal (padre biológico, padrastro, tío, abuelo). Cuidador primario madre, medida a través de la pregunta: ¿durante la mayor parte de su vida el rol de su madre lo ha cumplido?, de tipo politómica nominal (madre biológica, madrastra, tia, abuela); sexo, dicotómica nominal (femenino y masculino); edad (numérica discreta); especialidad, politómica nominal (Ciencias de la Salud, Ingeniería, Derecho y ciencias políticas, Ciencias de la educación y humanidades, Ciencias económicas y comerciales); lugar de nacimiento del estudiante, politómica nominal (costa, sierra, selva, extranjero); distrito, politómica nominal (Lima norte, Lima centro, Lima sur, Lima provincia); estado civil, politómica nominal (soltero, casado conviviente, divorciado, viudo); tipo de familia, definido como la estructura y relación de parentesco entre los integrantes y los lazos sanguíneos dentro del hogar (13), politómica nominal (nuclear, monoparental, compuesta y extensa); religión, politómica nominal (católica, no católica, ateo); trabajo, politómica nominal (sí, no).

Para el análisis de datos se utilizó el software estadístico STATA versión 14, se empleó el Chi cuadrado en el análisis de las variables categóricas y U de Mann Whitney para las variables numéricas (no paramétricas) y categóricas. Considerando para la significancia estadística un valor menor o igual a 0.05. Asimismo, este estudio fue aprobado por el Comité de Ética de la Universidad Católica Sedes Sapientiae.

\section{RESULTADOS}

De los 187 encuestados, la edad promedio fue de 18.7 años, el $61 \%$ fueron mujeres, el $96 \%$ eran solteros, el $83 \%$ no trabajaba, el $57 \%$ proveniente de una familia nuclear al momento de la encuesta. Mientras que el $90 \%$ tuvo a su madre biológica y el $76 \%$ al padre biológico como cuidadores primarios. Entre los estudiantes en la dimensión cuidado se observa un nivel bajo tanto en la madre (98.9\%) como en el padre (93.6\%) y en la dimensión sobreprotección se observa un nivel alto tanto en el padre (82.3\%) como en la madre $(93.0 \%)$. El resto de los resultados se puede observar en la tabla 1.

Tabla 1. Descripción de los factores sociodemográficos

\begin{tabular}{lcc}
\hline Sexo & n & \% \\
$\quad$ Masculino & 72 & 38.5 \\
$\quad$ Femenino & 115 & 61.5 \\
Edad (m \pm DS) & $18.7 \pm 4.6$ \\
Especialidad & \multicolumn{2}{c}{} \\
$\quad$ Ciencias de la salud & 52 & 28.0 \\
$\quad$ Ingeniería & 40 & 21.4 \\
$\quad$ Derecho y ciencias políticas & 17 & 9.1 \\
$\quad$ Ciencias de la educación y & 39 & 21.0 \\
$\quad$ humanidades & & \\
$\quad$ Ciencias económicas y comerciales & 39 & 21.0 \\
Lugar de nacimiento & & \\
$\quad$ Costa & 157 & 84.0 \\
$\quad$ Sierra & 23 & 12.3 \\
$\quad$ Selva & 3 & 2.0 \\
Distrito & & \\
$\quad$ Lima Norte & 147 & 78.6 \\
$\quad$ Lima Centro & 28 & 15.0 \\
$\quad$ Lima Sur & 3 & 2.0 \\
Lima provincia & 9 & 5.0 \\
\hline
\end{tabular}


Tabla 1. continúa

\begin{tabular}{|c|c|c|}
\hline & $\mathbf{n}$ & $\%$ \\
\hline \multicolumn{3}{|l|}{ Estado civil } \\
\hline Soltero & 180 & 96.2 \\
\hline Casado & 4 & 2.1 \\
\hline Conviviente & 3 & 2.0 \\
\hline \multicolumn{3}{|l|}{ Trabajo } \\
\hline Sí trabaja & 31 & 17.7 \\
\hline No trabaja & 156 & 83.4 \\
\hline \multicolumn{3}{|l|}{ Tipo de familia } \\
\hline Nuclear & 107 & 57.2 \\
\hline Uniparental & 30 & 16.0 \\
\hline Compuesta & 13 & 7.0 \\
\hline Extensa & 37 & 19.8 \\
\hline \multicolumn{3}{|l|}{ Religión } \\
\hline Católica & 150 & 80.2 \\
\hline No católica & 21 & 11.2 \\
\hline \multicolumn{3}{|c|}{ Cuidador primario padre } \\
\hline Padre biológico & 142 & 76.0 \\
\hline Padrastro & 8 & 4.2 \\
\hline Tío & 6 & 3.2 \\
\hline Abuelo & 18 & 9.7 \\
\hline Hermanos & 5 & 2.7 \\
\hline \multicolumn{3}{|c|}{ Cuidador primario madre } \\
\hline Madre biológica & 169 & 90.3 \\
\hline Madrastra & 1 & 0.5 \\
\hline Tía & 2 & 1.1 \\
\hline Abuela & 14 & 7.4 \\
\hline \multicolumn{3}{|l|}{ Cuidado madre } \\
\hline Bajo & 185 & 98.9 \\
\hline Alto & 2 & 1.4 \\
\hline \multicolumn{3}{|l|}{ Cuidado padre } \\
\hline Bajo & 175 & 93.6 \\
\hline Alto & 12 & 6.4 \\
\hline \multicolumn{3}{|l|}{ Sobreprotección madre } \\
\hline Bajo & 14 & 7.5 \\
\hline Alto & 173 & 92.5 \\
\hline \multicolumn{3}{|l|}{ Sobreprotección padre } \\
\hline Bajo & 28 & 17.7 \\
\hline Alto & 173 & 82.3 \\
\hline
\end{tabular}

En la tabla 2 se observa que existe relación significativa entre la dimensión de sobreprotección padre $(\mathrm{p}=0.00)$ y sobreprotección madre $(\mathrm{p}=0.008)$ y el cuidador primario. Asimismo, se registró un mayor porcentaje de la dimensión sobreprotección, tanto en la madre (93.5\%) como en el padre $(88.7 \%)$.

Existe relación significativa entre la dimensión de sobreprotección madre con el lugar de nacimiento $(\mathrm{p}=0.001)$. Se observan valores altos de sobreprotección madre en la región Costa (93.7\%) y Sierra $(95.7 \%)$. Ver tabla 3.

\section{DISCUSIÓN}

En el estudio se encontró relación significativa entre el ser cuidador primario y la actitud de sobreprotección mostrando los valores más altos en las categorías de cuidador primario padre y madre biológicos. Así mismo, el lugar de nacimiento se relaciona con la sobreprotección, encontrándose altos valores en la cuidadora primaria madre y la Región de la Costa y la Sierra.

Los padres biológicos son percibidos como más sobreprotectores que otros cuidadores primarios. Diversos estudios confirman lo hallado $(2,5,14)$. Se refiere que el padre al cuidar a los hijos suele ejercer un estilo de crianza de control para protegerlos de situaciones de riesgo que puedan rodear al adolescente. Asimismo, el padre puede utilizar prácticas orientadas al objeto que los hijos desean o mediante la expresión de amor hacia ellos (15). Es decir, el padre puede condicionar respuestas de conductas del adolescente al comprarle o brindarle un objeto que le interesa al hijo para interferir en sus decisiones, lo cual conlleva a la sobreprotección (15). Por otro lado, puede existir una "triada asistidora" en la cual los padres para evitar separarse como pareja, utilizan al hijo de unión para negar su problema (16). Esto conlleva a la sobreprotección del hijo donde crean diversas excusas (cuidado exagerado, ver al niño como enfermo y que requiere de atención). Además, los padres que brindan cuidados exagerados hacia sus hijos los exponen a un estilo de crianza que posteriormente podría ser imitado (7).

Se encontró asociación entre la sobreprotección de la madre y el lugar de nacimiento. Al respecto un estudio en el Perú reportó que el alto grado de control y el afecto desmedido se presentaron en el estilo parental de las madres, lo cual podría conllevar a riesgos en el desarrollo social del adolescente, manifestando mayor inhibición social, inseguridad y dificultad en la toma de decisiones (17) Por otra parte, un estudio reportó que los adolescentes de la selva perciben un estilo parental autoritativo caracterizado por padres que ejercen elevado control pero a la vez flexibilidad permitiendo una mayor independencia y 
Tabla 2. Asociación descriptiva entre las dimensiones de lazos parentales y cuidador primario

\begin{tabular}{|c|c|c|c|c|c|c|c|c|c|c|c|c|}
\hline & \multicolumn{3}{|c|}{$\begin{array}{c}\text { Sobreprotección } \\
\text { padre }\end{array}$} & \multicolumn{3}{|c|}{$\begin{array}{c}\text { Sobreprotección } \\
\text { madre }\end{array}$} & \multicolumn{3}{|c|}{ Cuidado padre } & \multicolumn{3}{|c|}{ Cuidado madre } \\
\hline & $\begin{array}{c}\text { Bajo } \\
\%\end{array}$ & $\begin{array}{c}\text { Alto } \\
\%\end{array}$ & $\mathbf{p}$ & $\begin{array}{c}\text { Bajo } \\
\%\end{array}$ & $\begin{array}{c}\text { Alto } \\
\%\end{array}$ & $\mathbf{p}$ & $\begin{array}{c}\text { Bajo } \\
\%\end{array}$ & $\begin{array}{c}\text { Alto } \\
\%\end{array}$ & $\mathbf{p}$ & $\begin{array}{c}\text { Bajo } \\
\%\end{array}$ & $\begin{array}{c}\text { Alto } \\
\%\end{array}$ & $\mathbf{p}$ \\
\hline $\begin{array}{l}\text { Cuidador primario } \\
\text { padre }\end{array}$ & & & 0.000 & & & 0.008 & & & 0.663 & & & 0.259 \\
\hline Padre biológico* & 11.27 & 88.7 & & 6.51 & 93.5 & & 94.4 & 5.7 & & 99.4 & 0.6 & \\
\hline Padrastro* & 37.5 & 62.5 & & 100 & 0 & & 100 & 0 & & 100 & 0 & \\
\hline Tio (a) & 50 & 50 & & 0 & 100 & & 83.3 & 16.7 & & 100 & 0 & \\
\hline Abuelo (a) & 22.2 & 77.8 & & 7.14 & 92.9 & & 88.9 & 11.1 & & 93.0 & 7.1 & \\
\hline Hermano (a) & 40 & 60 & & & & & 100 & 0 & & & & \\
\hline
\end{tabular}

*Padre biológico/madre biológica. Padrastro/madrastra

Tabla 3. Asociación descriptiva entre las dimensiones de lazos parentales y lugar de nacimiento

\begin{tabular}{|c|c|c|c|c|c|c|c|c|c|c|c|c|}
\hline & \multicolumn{3}{|c|}{$\begin{array}{c}\text { Sobreprotección } \\
\text { padre }\end{array}$} & \multicolumn{3}{|c|}{$\begin{array}{c}\text { Sobreprotección } \\
\text { madre }\end{array}$} & \multicolumn{3}{|c|}{ Cuidado padre } & \multicolumn{3}{|c|}{ Cuidado madre } \\
\hline & $\begin{array}{c}\text { Bajo } \\
\%\end{array}$ & $\begin{array}{c}\text { Alto } \\
\%\end{array}$ & $\mathbf{p}$ & $\begin{array}{c}\text { Bajo } \\
\%\end{array}$ & $\begin{array}{c}\text { Alto } \\
\%\end{array}$ & p & $\begin{array}{c}\text { Bajo } \\
\%\end{array}$ & $\begin{array}{c}\text { Alto } \\
\%\end{array}$ & p & $\begin{array}{c}\text { Bajo } \\
\%\end{array}$ & $\begin{array}{c}\text { Alto } \\
\%\end{array}$ & p \\
\hline $\begin{array}{l}\text { Lugar de } \\
\text { nacimiento }\end{array}$ & & & 0.665 & & & 0.001 & & & 0.200 & & & 0.943 \\
\hline Costa & 18.4 & 81.6 & & 6.4 & 93.7 & & 95.0 & 5.1 & & 98.8 & 1.2 & \\
\hline Sierra & 17.3 & 82.7 & & 4.4 & 95.7 & & 87.0 & 13.0 & & 100 & 0 & \\
\hline Selva & 0 & 100 & & 66.7 & 33.3 & & 100 & 0 & & 100 & 0 & \\
\hline
\end{tabular}

capacidades de comunicación y adaptación en los hijos (18). Estos hallazgos corroboran los altos niveles de sobreprotección encontrados en la región de la Costa y Sierra, puesto que los lazos parentales pueden variar en función del contexto sociocultural e incluso del medio geográfico.

Entre las limitaciones del estudio se debe señalar que no se tomaron en cuenta las siguientes variables: el tiempo de interacción entre el cuidador primario y el hijo; el nivel académico e ingreso percibido de los padres. Las mismas pudieran estar relacionadas con los vínculos parentales. Por otro lado, el sesgo de memoria, la temporalidad y la deseabilidad social pueden haber condicionado las respuestas. Sin embargo, cabe señalar que la consideración de las variables sociodemográficas conectadas al fenómeno es amplia siendo escasos los estudios peruanos que profundizan en las mismas.

\section{CONCLUSIONES}

A partir de los hallazgos los padres biológicos son percibidos como más sobreprotectores que otros cuidadores primarios. Esta relación indica de que si bien hay un vínculo estrecho, en algunos casos, este podría disminuir la autonomía, la capacidad de decisión y adaptación, efectos característicos de la sobreprotección.

En cuanto a la relación entre la sobreprotección de la madre y el lugar de nacimiento, es necesario considerar los factores psicosociales y culturales de las regiones que podrían influir en la manera de criar y proteger a los hijos. Esto con el fin de comprender la dinámica familiar y contribuir al desarrollo biopsicosocial del adolescente. Esta característica del lazo parental madre y sus efectos ya mencionados pueden prevenirse a través de escuelas para madres (sin exceptuar a los padres) en las cuales a través de técnicas cognitivoconductuales se les brinde herramientas que promuevan lazos parentales con niveles productivos de cuidado.

Es importante que los padres (o personas encargadas directamente de los niños) diferencien el cuidado adecuado de la sobreprotección hacia sus hijos, ya que podrían originar dificultades de interacción y adecuación al entorno social. Se sugiere promover la intervención psicológica sistemática para padres e hijos en el ámbito comunitario y educativo a través de talleres preventivos que incluyan como temas centrales el clima y la dinámica familiar, los estilos de comunicación y los lazos parentales adecuados. 


\section{REFERENCIAS BIBLIOGRÁFICAS}

1. Matalinares M, Díaz G. Influencia 7. Baumrind D. The influence of de los estilos parentales en la adicción al Internet en alumnos de secundaria del Perú. Revista de investigación en psicología. 2013;16(2):195-220.

2. Iglesias B, Romero E. Estilos parentales percibidos, psicopatología y personalidad en la adolescencia. Revista de Psicopatología y Psicología Clínica. 2009;14(1):63-77.

3. Inoñán K, Menor, M. Estilos de apego parental y dependencia emocional en las relaciones de pareja en jóvenes estudiantes de una universidad privada [Tesis licenciatura]. Chiclayo: Universidad privada Juan Mejía Baca;2014.

4. Parker G, Tupling H, Brown L. A parental bonding instrument. British Journal of Medical Psychology. 1979;52(1):1-10.

5. Sanchis F. Apego, acontecimientos vitales $\mathrm{y}$ depresión en una muestra de adolescentes [Tesis doctoral]. Barcelona: Universitat Ramon Llull;2008.

6. Gómez Y, Vallejo V, Villada J, Zambrano R. Caracterización de lazos parentales en estudiantes de pregrado de la Universidad de Antioquia. Revista de psicología universidad de Antioquia. 2009;1(1):35-53 parenting style on adolescent competence and substance use. Journal of Early Adolescence. 1991;11(1):56-95.

8. Krishnakumar A. Buehler C. Interparental conflict and parenting behaviors: A metaanalyticreview. Family Relations. 2000;49(1):25-44.

9. Enrique G, Fuentes M, García F. Barrios de riesgo, estilos de socialización parental y problemas de conducta en adolescentes. Psychosocial Intervention. 2010;19(3):265-278.

10. Instituto Nacional de Salud Mental. Estudio epidemiológico de salud mental en Lima Metropolitana y Callao-replicación 2012 [internet]. Lima: INSM; 2013. Disponible en: http://www.insm.gob.pe/investigac ion/archivos/estudios/2012\%20AS M\%20-EESM\%20-LM.pdf

11. Vásquez A, León, F. Relación entre los lazos parentales de la infancia y la satisfacción de las necesidades psicológicas básicas en un grupo de estudiantes de psicología de una universidad privada de Lima Metropolitana [Tesis licenciatura]. Lima: Universidad Peruana de Ciencias Aplicadas (UPC);2017.

12. Galarreta V. Propiedades psicométricas del Instrumento de Lazos Parentales en estudiantes de institutos superiores tecnológicos de la ciudad de Trujillo [Tesis licenciatura]. Trujillo: Universidad César Vallejo;2016.

13. Gutiérrez R, Díaz K, Román P. El concepto de familia en México: una revisión desde la mirada antropológica y demográfica. CIENCIA ergo-sum. 2016;23(3):219-228.

14. Abad C, Valle W. Relación entre lazos parentales y niveles de resentimiento en consumidores de sustancias psicoactivas, Chimbote, 2015. In Crescendo Ciencias de la salud. 2015;2(2):365-373.

15. Olivo D. Ansiedad y Estilos Parentales en un Grupo de Adolescentes de Lima Metropolitana [Tesis licenciatura]. Lima: Pontificia Universidad Católica del Perú;2012.

16. Minuchin S, Fishman H. Técnicas de terapia familiar. 1a ed. Buenos Aires: Paidós;2004.

17. Gutiérrez F, Huillca M. Estilos parentales y resiliencia global ante las adversidades en adolescentes. C. S. Independencia. Alto selva alegre. Arequipa - 2016 [Tesis licenciatura]. Arequipa: Universidad Nacional de San Agustín de Arequipa;2016.

18. López L, Huamaní, M. Estilos de crianza parental y problemas de conducta en adolescentes de una I.E. pública de Lima este, 2016. [Tesis licenciatura]. Lima: Universidad Peruana Unión;2017. 